\title{
ANFOTERICINA B NO TRATAMENTO DA NEUROCRIPTOCOCOSE EM PACIENTES SUBMETIDOS A TRANSPLANTE RENAL
}

\author{
J. P. S. NÓBREGA :
}

\begin{abstract}
RESUMO - Foram estudados 35 casos de neurocriptococose, 17 dos quais em pacientes submetidos a transplante renal. O objetivo foi avaliar a resposta terapêtica observada no grupo de pacientes transplantados renais com neurocriptococose, com ênfase à função renal, submetidos a esquema. medicamentoso que inclui a anfotericina $\mathbf{B}$. A resposta observada foi comparada aos resultados obtidos no grupo dos demaís pacientes com a mesma infecção, submetidos ao mesmo esquema medicamentoso, porém sem insuficiência renal prévia. Dos 35 pacientes, 10 faleceram nos primeiros dias do tratamento e, assim, 25 foram efetivamente tratados para neurocriptococose. Destes 25, 18 apresentavam outra condição clínica associada, sendo esta transplante renal em 15 deles, Os medicamentos utilizados basicamente foram a anfotericina $B$ intravenosa e intra-raqueana associada à 5-fluorocitosina via oral. Sete pacientes faleceram durante o trataniento. Assim. dos 35 pacientes que inicialmente compunham a casuistica, 17 faleceram $(48,57 \%)$ e 18 foram tratados com sucesso, nenhum deles apresentando recidiva. As intercorrências observadas durante o tratamento sāo discutidas e é feita correlação dos dados obtidos neste estudo aos disponiveis na literatura. A análise dos resultados obtidos permite ressaltar: a necessidade do surgimento de medicamentos mais eficazes e menos tóxicos para a terapêutica da neurocriptococose, sendo até o momento a anfotericina B o principal medicamento existente; o esquema terapêtico atualmente indicado consiste na associaçăo anfotericina $B+5$ fluorocitosina, havendo vantagens na utilizarāo simultânea da anfotericina $B$ pelas vias intravenosa e intra-raqueana. A análise dos resultados permite concluir por năo haver contra-indicaçăo ao uso da anfotericina $B$ por via intravenosa em transplantados renais com neurocriptococose.
\end{abstract}

\section{Amphotericin $B$ in the treatment of central nervous system cryptococcosis in patients submitted to renal transplantation.}

SUMMARY - Thirty-five cases of cryptococcosis of the central nervous system (CNS) were studied, 17 of them submitted to renal transplantation. The objective was to evaluate the therapeutic responses observed in the group of kidney transplant patients with CNS cryptococcosis. They were submitted to amphotericin $\mathbf{B}$ therapy, with emphasis to the renal function. The results in this group were compared with the outcome in the group of patients with the same infection, submitted to the same therapeutic scheme, but without previous impairment of renal function. Among the 35 patients, 20 were male; the age varied between 4 and 76 years. Associated clintcal conditions were noticed in 25 patients, 17 of them with renal transplantation. Among 35 patients, 10 died in the first days of the treatment; 25 patients were effectively treated for CNS cryptococcosis, 18 of them with associated clinical conditions; 15 were kidney transplant patients. The drugs used in the treatment of CNS cryptococcosis were, as possible, the amphotericin $B$ by intravenous and intrathecal route (Jumbar puncture) associated with 5-fluorocytosine. Seven patients died during the treatment; then, of the 35 patients who were initially evaluated, 17 died and 18 were successfully treated, with a death rate of $48.57 \%$. Various intercurrences were observed with the use of amphotericin $B$ and 5 -fluorocytosine. The clinical and therapeutic resuits recorded in this study were compared with the information met in literature. The

Clínica Neurológica do Hospital das Clínicas da Faculdade de Medicina da Universidade ao Departamento de Neurologia da FMUSP em 1987.

Centro de Investigacóes em Neurologia, FMUSP - Caixa Postal 5199 - 01051 São Paulo SP - Brasil. 
analysis of the results emphasizes the need of the discovery of better and less toxic drugs than those currently used. Amphotericin $\mathbf{B}$ still is the most important drug in the treatment of CNS cryptococcosis and the therapeutic scheme currently recommended consists in the association of amphotericin $\mathbf{B}$ and $\mathbf{5 - f l u o r o c y t o s i n e , ~ a n d ~ t h e r e ~ h a s ~ b e e n ~ a l s o ~ a d v a n t a g e ~ i n ~}$ the simultaneous use of intravenous and intrathecal amphotericin $B$. Statistical analysis of the results showed that there is no harm with the use of intravenous amphotericin $B$ in renal transplanted patients with cryptococcosis of the central nervous system.

O comprometimento do sistema nervoso central (SNC) pelo Cryptococcus neoformans, agente da criptococose, constituti capitulo importante dentre os processos infecciosos que acometem o SNC e seus envoltórios. Tal fato decorre de uma série de circunstâncias dentre as quais merecem ser destacadas: a gravidade da neurocriptococose, a maioria absoluta dos pacientes evoluindo para óbito ou sequelas importantes se não forem tratados precoce e adequadamente; inexistência, até o momento, de drogas realmente eficazes e que sejam desprovidas de efeitos tóxicos importantes, muitas vezes limitantes de sua utilizaçāo; aumento na incidência da criptococose, fato este decorrente, em grande parte, do número crescente de pacientes submetidos a condutas terapêtuticas que favorecem a transformaçāo de agentes oportunistas em patogênicos ou, como no caso do Cryptococcus neofarmans, favorecem que agentes potencialmente patogênicos, convivendo em simbiose com o hospedeiro, em determinado momento, oportunisticamente, rompam este equilibrio e desencadejem a doença 7,9,10,26,30,36,49. É bem estabelecido que determinados grupos de pacientes são mais susceptiveis a infecções em decorrência de imunossupressão causada pela própria doença e ou pelas drogas utilizadas em seu tratamento. Transplantados renais mantidos cronicamente com drogas imunossupressoras e corticóides constituem exemplo altamente significativo quanto a esses aspectos 14,19,20,22,34,35,39. O Cryptococcus neoforinans apresenta intenso tropismo pelo SNC, sendo a neurocriptococose a infecção micótica mais freqüente do SNC e de seus envoltórios 16,17,19,22,25. A manipulação terapêutica da neurocriptococose é difícil e prolongada, exigindo avaliação constante do paciente devido às inúmeras intercorrências que podem surgir, decorrentes seja da gravidade da doença, seja da toxicidade dos medicamentos utilızados em seu tratamento. Poucos são os medicamentos com real indicação no tratamento desta micose, porém, inúmeros e controvertidos são os esquemas terapêuticos propostos. A droga de escolha é a anfotericina B (ANFO-B) cujos efeitos nefrotóxicos são amplamente conhecidos $2,5,8,19,21,28,30$; tal fato faz com que haja receio de sua utilização em pacientes com função renal comprometida e, em especial, em transplantados renais tendo em vista eventual risco de perda do rim transplantado $6,7,13,18,25,35,36,40,42$.

Neste estudo a ANFO B foi utilizada sistematicamente em pacientes transplantados renais com neurocriptococose; tal conduta partiu da premissa de que a ANFO-B realmente é a droga de escolha no tratamento desta micose, doença de evolução invariavelmente fatal se não tratada adequadamente. Não a utilizar com o argumento da necessidade de preservar o rim transplantado corresponde a condenar o paciente à morte, obviamente com a perda do transplante. Uma vida náo se refaz, um transplante sim. É propósito deste trabalho estudar a resposta terapêtica observada em um grupo de pacientes transplantados renais com neurocriptococose, com ênfase à funçăo renal, submetidos a esquema medicamentoso que inclui a ANFO-B e compará-la aos resultados obtidos em um grupo de pacientes com a mesma infeç̧ão, submetidos ao mesmo esquema medicamentoso, porém, sem apresentar prévio comprometimento da função renal.

\section{CASUISTICA E METODOS}

Foram estudados 35 casos de neurocriptococose, 17 dos quais em pacientes submetiaus a transplante renal, todos acompanhados pessoalmente pelo autor em período compreendido de janeiro de 1972 a dezembro de 1983, a maioria das observaçoses sendo feitas na Clínica Neurológica do Hospital das Clínicas da FMUSP. Todos os transplantados pertenciam a Unidade de Transplante Renal do mesmo Hospital. Todos tinham diagnóstico de certeza de neurocriptococose, estabelecido por exame do LCR em 34 pacientes e por procedimento neurocirúrgico em um. Dos 35 pacientes, 20 pertencem ao sexo masculino e 15 ao feminino, a idade variando de 4 a 76 anos. Na tabela 1 estão relacionados os 35 pacientes e as 
respectivas condiçóes clínicas associadas, existentes em 25 pacientes, em 17 dos quais era transplante renal. Dos 35 pacientes estudados, 10 faleceram nos primeiros dias de tratamento e por este motivo não serão avaliados em relaçåo às drogas utilizadas, tendo em vista o tempo insuficiente de tratamento. Na tabela 1 estão indicados os 25 pacientes que foram efetivamente tratados; estes 25 pacientes servirão de base para as consideraçōes que serão desenvolvidas em relação ao tratamento da neurocriptococose.

Os medicamentos utilizados foram basicamente a ANFO-B e a 5-fluorocitosina (5-FC), sempre que possivel em associacão. A ANFO-B, sempre que possivel, foi administrada con-

\begin{tabular}{|c|c|c|c|}
\hline Caso & Nome & Condição associada & $\begin{array}{c}\text { Efetivamente } \\
\text { tratado }\end{array}$ \\
\hline 1 & $\mathrm{JCZ}$ & & \\
\hline 2 & JDSB & & + \\
\hline 3 & FNPN & Transplante renal & + \\
\hline 4 & ATO & Tuberculose & \\
\hline 5 & LMBM & Transplante renal & + \\
\hline 6 & LRFS & & + \\
\hline 7 & BJS & Tuberculose & \\
\hline 8 & ILO & Transplante renal & + \\
\hline 9 & $\mathbf{A M}$ & Adenocarcinoma de útero & + \\
\hline 10 & Y'T & Transplante renal & + \\
\hline 11 & JFR & & \\
\hline 12 & ASS & & + \\
\hline 13 & $\mathrm{MHBC}$ & Transplante renal & + \\
\hline 14 & $\mathrm{AC}$ & Transplante renal & + \\
\hline 15 & ML & Diabetes melito & + \\
\hline 16 & MAPA & Transplante renal & $t$ \\
\hline 17 & $\mathbf{A P A}$ & Leucemia linfóide crônica & \\
\hline 18 & ABA & Transplante renal & + \\
\hline 19 & LAG & Transplante renal & + \\
\hline 20 & JRA & & + \\
\hline 21 & $\mathbf{J G}$ & Transplante renal & + \\
\hline 22 & $\mathrm{AD}$ & Cirrose hepatica & \\
\hline 23 & $\mathrm{SN}$ & Transplante renal & \\
\hline 24 & CFBN & Transplante renal & + \\
\hline 25 & J RM & & + \\
\hline 26 & JPB & & + \\
\hline 27 & $\mathrm{TS}$ & Hiperparatireoidismo terciário & \\
\hline 28 & NHA & Linfoma & + \\
\hline 29 & SSS & & \\
\hline 30 & MLP & Transplante renal & + \\
\hline 31 & GFS & & + \\
\hline 32 & $\mathrm{SHM}$ & Transplante renal & + \\
\hline 33 & MLF & Transplante renal & \\
\hline 34 & JMT & Transplante renal & + \\
\hline 35 & $\mathrm{CM}$ & Transplante renal & + \\
\hline Total & 35 & 25 & 25 \\
\hline
\end{tabular}

Tabela 1 - Neurocriptococose: nome dos pacientes (iniciais); condiçoses clinicas a ela associadas (condicĩo associada); indicagäo ( + ) dos casos que receberam tratamento especifico em dosagem suficiente (efetivamente tratado). 
juntamente pelas vias intravenosa (IV) e intra-raqueana lombar (IR); em 5 pacientes foi também utilizada por via intraventricular, através da câmara de Rickham. Esta última via não foi mais utilizada tendo em vista a ocorrência de complicaçōes intensas e de propiciar benefícios discutiveis, Dois pacientes nảo receberam ANFO-B IV, um não recebeu ANFO-B IR e dois não receberam 5-FC. Em relaçāo à ANFO-B IV não foi possivel fixar dose diária constante para o tratamento tendo em vista ela depender da tolerância de cada paciente e a sua manutenção, da tolerância renal. Como regra o tratamento era iniciado com doses baixas, 5 a $10 / \mathrm{mg} /$ dia, as quais eram progressivamente aumentadas até atingir-se um máximo de $1 \mathrm{mg} / \mathrm{Kg} /$ dia ou $50 \mathrm{mg} /$ dia. Em pacientes transplantados renais inicialmente foram utilizadas doses menores, porém, frente aos primeiros resultados obtidos, posteriormente as doses foram as mesmas utilizadas em pacientes não transplantados. A partir do caso 24 a dose diária da ANFO-B IV foi reduzida, atingindo um máximo de $0,5 \mathrm{mg} / \mathrm{Kg} / \mathrm{dia} \mathrm{cm}$ todos os pacientes, independentemente da existència ou não de transplante renal; a partir deste paciente é que foi adotado o esquema terapêtico proposto por Medoff \& col. (28) e que consiste na associação da ANFO-B IV em doses baixas à 5 -FC em doses convencionais. As infusōes IV da ANFO-B eram diárias, adotando-se regime de dias alternados apenas como última alternativa para atenuar intercorréncias tóxicas decorrentes do uso da ANFO-B IV. A administraça da ANFO-B IR era iniciada com dose de 0,1mg para testar a sensibilidade do paciente, sendo então progressivamente aumentada até $1 \mathrm{mg}$ por aplicaçāo, sempre associadà à hidrocortisona na tentativa de evitar ou minorar efeitos colaterais; a via JR, como regra, era utilizada em dias alternados, três vezes por semana. A 5-FC foi utilizađa na dose de 150 a $200 \mathrm{mg} / \mathrm{Kg} /$ dia, por via oral, em 4 tomadas, os intervalos entre as doses, em pacientes com insuficiência renal, sendo dádo pela depuração de creatinina, segundo esquema proposto por Schonebeck \& col. (3\%). Em relaçāo à toxicidade da ANFO-B foi dada ênfase à nefrotoxicidade e à hipopotassemia; a função renal foi monitorizada por dosagens periódicas de uréia, creatinina e da depuração de creatinina. Alteraçōes consideradas significativas destes parâmetros, principaimente da crcatinina e da depuraçăo, eram seguidas, inicialmente, de diminuiçāo da dose diária ca ANFO-B e, se necessário, de administraçāo em dias alternados. Ocorrendo normalização desses exames, a dose diária retornava aos valores anteriores; caso contrário era mantida nos valores em que os dados laboratoriais se mantivessem em niveis considerados adequados para a função renal. Alteração persistente da função renal era acompanhada da suspensão da utilização da ANFO-B por via IV. A hipopotassemia foi corrigida com administração oral de potássio €, se necessáría, com administração IV; caso não fosse corrigida com estes procedimentos, era seguidొ $o$ esquema utilizado em relação à nefrotoxicldade. As intercorrências verificadas com a utilização da via IR eram seguidas da interrupçāo temporấria ou definitiva de sua utilização, dependendo da intensidade e do tipo de intercorrência. Em relaçẩo à toxicidade da 5 -FC as atenções se voltaram basicamente para as alteraçōes hematológicas, as quais foram avaliadas no minimo duas vezes por semana; alteracōes hematológicas significativas e persistentes eram seguidas da suspensão definitiva da administraçāo da 5-FC. Não foram estabelecidos limites quanto à dose total de ANFO-B IV e IR, nem quanto à duração do tratamento. O critério de suspensão do tratamento foi basicamente laboratorial, já que clinicamente o paciente pode tornar-se assintomático em fase precoce da terapèutica. $O$ critério adotado foi o da existência de no mínimo três culturas de LCR sucessivamente negativas, cada cultura sendo observada durante um mês, mesmo na presença de exames da tinta da China positivos. Considcrou-se critério de cura se, clínica e laboratorialmente, não houvesse intercorrências no prazo de um ano após o término do tratamento, periodo este em que costuma ocorrer a maioria das recidivas. Neste espaço de tempo as culturas do LCR devem ser persistentemente negativas, embora o exame da tinta da China possa não o ser.

A avaliação estatística dos resultados obtidos, estudo das freqüências de intercorrências verificadas com o uso da ANFO-B por via IV em pacientes transplantados renais relativamente aos não transplantados, foi efetuada utilizando o teste exato đe Fisher, adimitindo alf $a=0,1$.

\section{RESULTADOS}

Dos 25 pacientes avaliados quanto aos objetivos deste cstudo, os dados de presença ou não de condiçāo clínica associada, drogas utilizadas e respectivas vias de administração estão relacionados na tabela 2 . Em 18 pacientes dos 25 efetivamente tratados foi reconhecido haver condição clínica associada, 15 deles tendo sido submetidos a transplante renal. Na tabela 3 estão relacionadas as drogas utilizadas no tratamento da neurocriptococose, o número total de pacientes que utilizou cada uma delas em suas diferentes vias de administração, o número de intercorrências com cada uma delas, o número de pacientes trans- 
Tratamento

\begin{tabular}{|c|c|c|c|c|c|c|c|c|}
\hline \multirow[t]{2}{*}{ Caso } & \multicolumn{3}{|c|}{ Anfo-B } & \multirow[t]{2}{*}{ 5-FC } & \multicolumn{2}{|c|}{ Miconazole } & \multirow[t]{2}{*}{ Cond. } & \multirow[t]{2}{*}{ assoc. } \\
\hline & IV & IR & $\mathrm{V}$ & & IV & IR & & \\
\hline 2 & + & + & -+ & + & & & & \\
\hline 3 & + & $+t$ & & + & & & $\dot{+}$ & $T R$ \\
\hline 5 & + & + & & + & & & + & TR \\
\hline 6 & $t$ & tr & & $t$ & & & & \\
\hline 8 & & + & & + & & & + & $\mathrm{TR}$ \\
\hline 9 & + & $t$ & + & + & & & + & \\
\hline 10 & & + & + & + & & & + & $T R$ \\
\hline 12 & + & + & + & + & & & & \\
\hline 13 & + & + & + & + & & & + & $\mathrm{TR}$ \\
\hline 14 & + & + & & + & & & + & $T R$ \\
\hline 15 & + & + & & + & & & + & \\
\hline 16 & + & + & & + & + & + & + & $\mathbf{T R}$ \\
\hline 18 & + & + & & + & + & + & + & TR \\
\hline 19 & + & + & & & & & + & $\mathbf{T R}$ \\
\hline 20 & + & + & & & & & & \\
\hline 21 & + & + & & + & & & + & $\mathbf{T R}$ \\
\hline 24 & + & + & & + & & & + & TR \\
\hline 25 & + & + & & + & & & & \\
\hline 26 & + & + & & + & & & & \\
\hline 28 & + & $\therefore$ & & + & & & + & \\
\hline 30 & + & + & & + & & & + & $\mathbf{T R}$ \\
\hline 31 & + & + & & + & & & & \\
\hline 32 & + & + & & + & & & + & $T R$ \\
\hline 34 & + & + & & + & & & + & $T R$ \\
\hline 35 & + & & & + & & & + & TR \\
\hline Total & 23 & 24 & 5 & 23 & 2 & 2 & 18 & 15 \\
\hline
\end{tabular}

Tabela 2 - Neurocriptococose: pacientes efetivamente tratados (25). Drogas $e$ vias usadas (Tratamento) $e$ condicóes clinicas asociadas (Cond. assoc.). Drogas utilizadas: anfotericina $B$ (Anfo-B), 5-fluorocitosina (5-FC), miconazole. Vias de administrasão (+): intravenosa (IV), intra-raqueana (IR) intraventricular (V); 5-FC administrado via oral. Cond. assoc.: presente ( + ), transplante renal (TR).

plantados renais que as utilizaram e o número de intercorrénciás verificađas nestes pacientes transplantados. Na tabela 4 estão relacionados os mesmos dados referidos na tabela 3 , porém, abrangendo até $o$ caso 23; até este paciente a ANFO-B foi utilizada em doses de até $1 \mathrm{mg} / \mathrm{Kg} / \mathrm{dia}$, associada ả $5 \cdot \mathrm{FC}$ em doses convencionais. Na tabela 5 estāo relacionados os mesmos dados referidos nas tabelas 3 e 4, porém, abrangendo os pacientes tratados a partir do caso 24 e nos quais a dose diária da ANFO-B não ultrapassou $0.5 \mathrm{mg} / \mathrm{Kg} / \mathrm{dia}$, associada à 5-FC em doses convencionaís.

Dos 25 pacientes efetivamente tratados, 7 faleceram no curso do tratamento, 5 sendo transplantados renais; nos demais, não ocorreram recidivas. No estudo das frcqü.ı̂ncias de intercorrências verificadas com o uso da ANFO-B por via IV em pacientes transplantados renais relativamente aos năo transpla.ntados (tabela 3), o teste exato de Fisher mostrou nitida diferença, estatisticamente significativa $(p=0,001)$, ou seja, os pacientes transplantudos renais apresentaram menos intercorrências do que os não transplantados. 


\begin{tabular}{|c|c|c|c|c|c|c|}
\hline Droga & $\begin{array}{l}\text { Total } \\
\text { Casos }\end{array}$ & $\begin{array}{l}\text { Total } \\
\text { interc. }\end{array}$ & $\begin{array}{c}\mathrm{N}^{\circ} \\
\text { nāo } \mathrm{TR}\end{array}$ & $\begin{array}{l}\text { Interc. } \\
\text { não TR }\end{array}$ & $\begin{array}{l}\text { No } \\
\text { TR }\end{array}$ & $\begin{array}{c}\text { Interc } \\
\mathrm{TR}\end{array}$ \\
\hline Anfo-B IV & 23 & 11 & 10 & 8 & 13 & 3 \\
\hline Anfo-B IR & 24 & 5 & 10 & 2 & 14 & 3 \\
\hline Anfo-B V & 5 & 5 & 3 & 3 & 2 & 2 \\
\hline 5-FC & 23 & 11 & 9 & 3 & 14 & 8 \\
\hline Miconazole & 2 & 0 & 0 & 0 & 2 & 0 \\
\hline
\end{tabular}

Tabcla 3-Drogas utilizadas $e$ intercorrências verificadas no total dos pacientes $e$ nos pacien tes com $e$ sem transplante renal. Legenda: No, número de casos; Interc., intercorrência; $T R$, transplante renal; Anfo- $B$, anfotericina $B ; 5-F C, 5$-fluorocitosina; $I V$, intravenoso; $I R$, intra-raqueano; $V$, intraventricular.

\begin{tabular}{|c|c|c|c|c|c|c|}
\hline Droga & $\begin{array}{l}\text { Total } \\
\text { Casos }\end{array}$ & $\begin{array}{l}\text { Total } \\
\text { interc. }\end{array}$ & $\begin{array}{c}\text { No } \\
\text { não TR }\end{array}$ & $\begin{array}{l}\text { Interc. } \\
\text { não TR }\end{array}$ & $\begin{array}{l}\text { No } \\
\text { TR }\end{array}$ & $\begin{array}{c}\text { Interc. } \\
\text { TR }\end{array}$ \\
\hline Anfo-B IV & 14 & 9 & 6 & 6 & 8 & 3 \\
\hline Anfo-B IR & 16 & 5 & 6 & 2 & 10 & 3 \\
\hline Anfo-B V & 5 & 5 & 3 & 3 & 2 & 2 \\
\hline 5-FC & 14 & 10 & 5 & 3 & 9 & 7 \\
\hline Miconazole & 2 & 0 & 0 & 0 & 2 & 0 \\
\hline
\end{tabular}

Tabela 4 - Drogas utilizadas e intercorrências verificadas até o caso 2s. Legenda: ver tabela 3.

\begin{tabular}{|c|c|c|c|c|c|c|}
\hline Droga & $\begin{array}{l}\text { Total } \\
\text { Casos }\end{array}$ & $\begin{array}{l}\text { Total } \\
\text { interc. }\end{array}$ & $\begin{array}{c}\text { No } \\
\text { não } T R\end{array}$ & $\begin{array}{l}\text { Interc. } \\
\text { não TRR }\end{array}$ & $\begin{array}{l}\text { No } \\
\text { TR }\end{array}$ & $\begin{array}{c}\text { Interc. } \\
\text { TR }\end{array}$ \\
\hline Anfo-B IV & 9 & 2 & 4 & 2 & 5 & 0 \\
\hline Anfo-B IR & 8 & 0 & 4 & 0 & 4 & 0 \\
\hline Anfo-B V & 0 & 0 & 0 & 0 & 0 & 0 \\
\hline $5-F C$ & 9 & 1 & 4 & 0 & 5 & 1 \\
\hline Miconazole & 0 & 0 & 0 & 0 & 0 & 0 \\
\hline
\end{tabular}

Tabela 5 - Drogas utilizadas e intercorrências verificadas a partir do caso 84. Legenda: ver tabela 3 .

\section{COMENTARIOS}

Dois são os medicamentos até o niomento comprovadamente eficazes no tratamento da neurocriptococose, ANFO-B e 5-FC, porém, inúmeros são os esquemas terapêuticos propostos, entre eles sobressaindo-se a associação ANFO-B e $j-\mathrm{FC}^{2}-5,11,14,21,34,33,44-46,48$. Devido a esta multiplicidade de esquemas, resolvemos adotar rotineiramente, desde que possivel, a associação ANFO-B e 5-FC, a ANFO-B sendo administrada simultaneamente pelas vias IV e IR, tendo em vista permear pobremente a barreira hematoencefálica. A opção pelo uso sistemático da via IR para a 
administração da ANFO-B decorreu da constatação de relatos de número elevado de recidivas, assim como da falta de critérios consistentes para a indicação de seu uso. O número elevado de óbitos ainda relatados na literatura e confirmado neste estudo $(48,57 \%)$ justifica plenamente adotar-se conduta terapêtica enérgica em todos os casos de neurocriptococose $1,11,14,15,19,36,40,41,44,46,48$. O esquema terapêtico adotado mostiou-se eficaz, pors $\mathrm{km}$ nenhum dos pacientes que evoluıram satisfatoriamente ocorreu recidiva.

A decisão de utilizar a associaçăo ANFO-B e 5-FC no tratamento de todos os casos de neurocriptococose esbarrou, inicialmente, no receio do emprego da ANFO-B IV em pacientes com patologia renal e entre os quais os transplantados renais constituem o exemplo mais significativo. Os receios fundamentavam-se basicamente na refrotoxicidade da ANFO-B e, em consequência, no eventual risco de perda do rim transplantado; posteriormente, à medida em que a associação ANFO-B e 5 FC firmou-se como esquema de escolha no tratamento da neurocriptococose, surgiu o receio de que a nefrotoxicidade da ANFO-B pudesse potencializar os efettos toxicos, principalmente hematológicos, da 5-FC e que esse fato fosse mais intenso em pacientes con atteraçōes renais $4,1 \$, 10,28,<9,34,35$. Dicidimos entāo que, face à gravidade da neurocriptococose e frente à importância do uso da ANFO-B em seu tratamento, seria preferivel correr o risco de eventual perda do transplante renal do que da vida do paciente. Esta nossa resolução baseou.se, também, em alguns relatos da literatura a respeito dos bons resultados obtidos no tratamento da neurocriptococose em transplantados renais, seja em relação à infecção, seja quanto à função renal17,19,22,23,32,33,38,43. O presente estudo foi entăo esquematizado com o objetivo específico de avaliar a resposta terapêutica observada em um grupo de pacientes transplantados renais com neurocriptococose, com ènfase à função renal, submetidos a esquema terapêutico que inclui a ANFO-B, utilizada por via IV e IR e a 5-FC, e compara-la ao: resultacios ovtidos em um grupo de pacientes com a mesma patologia, submetidos ao mesmo esquema medicamentoso, porém, sem insuficiência renal prévia.

Dos 35 pacientes que compõem a casuistica, 20 pertencem ao sexo masculino, ocorrendo então discreto predominio deste sexo, porém, não na proporçăo de 2:1 relatada na literatura; a idade variou de 4 a 76 anos, com nitido predominio entre a terceira e quinta décadas, fato este concorde ao citado na literatura 14-16,26,47. Dos 35 pacientes, em $25(71.42 \%)$ existiam condiçōes clinicas associadas, sendo que 17 eram transplantados renais; estes correspondem a $68 \%$ do total de pacientes com condição clínica associada à neurocriptococose. Esta maior incidência de condiçōes clínicas associadas em relaçāo aos dados da literatura (30 a $50 \%)$ está provavelmente relacionáda ao número elevado de pacientes transplantados renais nesta casuistica $16,26,40,47,49$. Ocorreram 17 óbitos $(48,57 \%)$, 10 em fases iniciais do tratamento e 7 no curso do tratamento; esses dados ressaltam a gravidade da neurocriptococose. Dos 17 pacientes que faleceram, em 12 havia presença de condição clinica associada, 7 sendo transplantados renais e 5 apresentando outras pátologias. Em transplantados renais, dois dos 7 óbitos ocorridos durante tratamento foram ocasionados por hipertensāo intrácraniana rebelde a todas as condutas adotadas, clinicas ou cirúrgicas; nos demais, foi devido a septicemia. A alta porcentagem de óbitos em pacientes com condição clínica associada à neurocriptococose $(70,58 \%)$ verificada em nossa casuística confirma os dados da literatura quanto ao pior prognóstico nesses casos. Este fato justifica também o elevado número de óbitos em nossa casuistica $(48,57 \%)$, valor acima dos citados em literatura, pois o número de pacientes com neurocriptococose e condiçöes clinicas asco iadas $(71,42 \%)$ também é superior ans valores citados na literatura $7,11,13,16,26,36,40,47,49$.

Assim, 25 pacientes foram submetidos a tratamento considerado adequado; a análise retrospectiva dos dois casos tratados com o miconazole nos levou a concluir pela não indicação de seu uso no tratamento da neurocriptococose. Da análise da tabela 3 verificamos que 11 pacientes apresentaram intercorrências à ANFO-B IV, 5 à ANFO-B IR e 11 à 5 FC. Tais intercorrências motivaram a suspensão definitiva de administração das drogas em apenas dois pacientes que receberam a ANFO-B IV e em todos os pacientes que receberam a ANFO-B IR e a 5-FC; todas essas intercorrências não acarretaram danos permanentes aos pacientes, ocorrendo reversão completa das alterações ocorridas com a suspensāo definitiva do medicamento e ou da via de sua administração, ou com a alteração de suas doses. As intecorrências observadas com a ANFO-B IV foram basicamente neufrotoxidade $e$ hipopotassemia $e$ com a 
5-FC, alteraçōes hematológicas; esses dados são concordes aos relatados em literatura $3,6,18,24,27,31,33,35,41,44,49$. Conclui-se, frente a estes resultados que a ANFO-B foi melhor tolerada que a $5-F C$, já que as intercorrências com a ANFO-B levaram a menor número de suspensões definitivas de seu uso.

Todos os pacientes que apresentaram intercorrências com o uso da 5-FC, e consequente interrupção de sua administração, ou apresentaram alterações renais com o uso concomitante da ANFO-B ou eram transplantados renais. Estes fatos são favoráveis à hipótese de que alteraçōes renais, mesmo discretas, possam acarretar aumento da concentração plasmática da $5-\mathrm{FC}$ e, consequentemente, aumento de sua toxicidade $3,5,12,24,37$. A análise do desdobramento da tabela 3 nas tabelas 4 e 5 , fornece dados a favor dessa hipótese. Assim, embora o número de casos em cada uma dessas tabelas seja pequeno e não passivel de análise estatística, verificamos que 10 das 11 intercorrências com a 5-FC ocorreram nos pacientes que constam da tabela 4 , ou seja, naqueles pacientes nos quais a ANFO-B foi utilizada em doses maiores que aquelas posteriormente preconizadas para a associação ANFO-B e 5-FC e adotadas em todos os pacientes que constam da tabela 5 . Nestes, em numero de 9 , tivemos apenas uma intercorrência com o uso da 5-FC, em paciente que tolerou bem a ANFO-B IV.

Dos 18 pacientes tratados com sucesso, 17 receberam ANFO-B IV, 17 receberam ANFO-B IR e 16 a 5-FC nas fases iniciais do tratamento; porém, devido às intercorrências verificadas, uma ou outra destas drogas e ou suas vias de administração foram suspensas definitivamente. Em conseqüência, durante todo o tratamento, 16 pacientes receberam ANFO-B IV, 14 receberam ANFO-B IR e 10 a 5-FC. Os esquemas terapêuticos utilizados ao final do tratamento destes 18 pacientes muitas vezes não foram os mesmos das fases iniciais. A ANFO-B foi a droga utilizada de maneira mais constante, confirmando os dados de literatura de que é o medicamento mais importante no tratamento da neurocriptococose $2,5,8,19,21,28,30$. A dose total de ANFO-B IV variou de 435 a $3675 \mathrm{mg}$ e a de ANFO-B IR, de 1,5 a $29 \mathrm{mg}$; o tempo de tratamento variou de 46 a 324 dias, com média de 116 dias, ou seja, aproximadamente 4 meses.

A análise estatística das frequências de intercorrências verificadas com o uso da ANFO-B por via IV em pacientes transplantados renais relativamente aos não transplantados, objetivo básico deste trabalho, revelou nítida diferença, estatisticamente significativa, mostrando que os pacientes transplantados renais com neurocriptococose reagem ao uso da ANFO-B IV no minimo tão bem como os pacientes com a mesma patologia mas com função renal conservada. Assim sendo é possivel concluir que não há contra-indicação ao uso da anfotericina $\mathrm{B}$, por via intravenosa, no tratamento da neurocriptococose em transplantados renais.

Conclusões - A análise dos resultados do tratamento a que foram submetidos 25 pacientes com neurocriptococose, 15 dos quais com transplante renal, permite as conclusões que se seguem, as duas últimas quanto ao objetivo básico deste estudo e as primeiras, de ordem geral. 1. Os medicamentos atualmente disponiveis para 0 tratamento da neurocriptococose nảo são os ideais, tendo em vista o elevado número de óbitos e de intercorrências por eles provocadas. 2. A anfotericina B é o principal medicamento existente até o momento para o tratamento da neurocriptococose. 3. O esquema terapêutico de escolha, atualmente, no tratamento da neurocriptococose consiste na associação anfotericina B e 5-fluorocitosina. 4. Quando da utilização da associação anfotericina B e 5-fluorocitosina, a anfotericina B deve ser empregada em doses menores que as habitualmente usádas se administrada isoladamente; isto para evitar efeitos tóxicos, principalmente hematológicos, decorrentes da utilização da 5-fluorocitosina, a qual deve ser administrada nas doses convencionais. 5. $O$ emprego simultâneo da anfotericina $\mathrm{B}$ pelas vias intravenosa e intra-raqueana, associada à 5-fluorocitosina, mostrou-se esquema terapêutico adequado, tendo em vista os resultados obtidos e a inexistência de recidivas. 6. As intercorrências secundárias ao tratamento observadas entre pacientes com transplante renal que desenvolveram neurocriptococose são de ordem a considerar que eles reagem ao uso da anfotericina $B$ no mínimo tăo bem como pacientes com neurocriptococose não submetidos a transplante renal. 7. Não está contra-indicada a utilização da anfotericina B por via intravenosa no tratamento da neurocriptococose em transplantados renais. 


\section{REFERENCIAS}

1. Atkinson AJ Jr, Bindschadler DD - Pharmacokinetics of intrathecally administered amphotericin B. Amer Rev Resp Dis 99:917, 1969.

2. Bell WE - Treatment of fungal infections of the central nervous system. Ann Neurol $9: 417,1981$.

3. Bennett JE - Flucytosine. Ann Intern Med 86:319, 1977.

4. Bennett JE, Dismukes WE, Duma RJ, Medofff G, Sande MA, Gallis H, Leonard J, Fields B'T, Bradshaw M, Haywood H, McGee ZA, Cate TR, Cobbs CG, Warner JF, Alling DW - A comparison of amphotericin B alone and combined with flucytosine in the treatment of cryptococcal meningitis. N Engl J Med 301:126, 1979.

5. Block ER, Bennett JE - The combined effect of 5-fluorocytosine and amphotericin B in the therapy of murine cryptococcosis (37049). Proc Soc Exp Biol 142:476, 1973.

6. Burgess JL, Birchall R - Nephrotoxicity of amphotericin B, with emphasis on changes in tubular function. Amer $J$ Med 53:77, 1972.

7. Butler WT, Alling DW, spickard A, Utz JP - Diagnostic and prognostic value of clinical and laboratory findings in cryptococcal meningitis: a follow-up study of forty patients. $N$ Engl J Med 270:59, 1964.

8. Butler WT, Bennett JE, Alling DW, Wertlake PT, Utz JP, Hill II GJ - Nephrotoxicity of amphotericin B: early and late effects in 81 patients. Ann Intern Med 61:175, 1964.

9. Campbell GD, Currier KD, Busey JF - Survival in untreated cryptococcal meningitis. Neurology $31: 1154,1981$.

10. Carton CA - Treatment of central nervous system cryptococcosis: a review and report of four cases treated with actidione. Ann Intern Med 37:123, 1952.

11. Codish SD, Tobias JS, Monaco AP - Recent advances in the treatment of systemic mycotic infections, Surg Gynec Obstet 148:435, 1979.

12. Dawborn JK, Page MD, Schiavone DJ - Use of 5-fluorocytosine in patients with impaired renal function. $\mathrm{Br}$ Med $J$ 4:382, 1973.

13. D:amond RD, Bennett JE - Prognostic factors in cryptococcal meningitis: a study in 111 cases. Ann Intern Med 80:176, 1974.

14. Dureux JB, Canton Ph, Neiman L, Etienne $Y$, Houppe JP - Cryptococcose neuroméningée: considérations diagnostiques et thérapeutiques: à propos de 5 observations personnelles et d'une revue de la littérature. Med Mal Infect 11:573, 1979.

15. Edwars VE, Sutherland JM, Tyrer $\mathrm{JH}_{\mathrm{H}}-$ Cryptococcosis of the central nervous system: epidemiological, clinical, and therapeutic features. J Neurol Neurosurg Psychiat 33:415, 1970.

16. Fetter BF, Filintworth GK, Hendry WS - Mycoses of the central nervous system. Williams \& Wilkins, Baltimore, 1967.

17. Gallis HA, Berman RA, Cate TR, Hamilton JD, Gunnelis JC, Stickel DL - Fungal infection following renal transplantation. Arch Intern Med 135:1163, 1975.

18. Goodman JS, Koenig MG - Amphotericin B: specifics of administration. Mod Treat $7: 581,1970$.

19. Gosseye-Lissoir F, Laterre E-C, Vandepitte J, Blaton H, Yourassowsky E, Schouttens E - Cryptococcose cérébroméningée: rôle favorisant de la thérapeutique immunosuppressive chez les transplantés rénaux. Path Biol 23:211, 1975.

20. Hart PD, Russell E Jr, Remington JS - The compromised host and infection: II. Deep fungal infection. $J$ Infect Dis 120:169, 1969.

21. Hermans PE, Keys TF - Antifungal agents used for deep-seated mycotic infections. Mayo Clin Proc 58:223, 1983.

22. Hooper DC, Pruitt AA, Rubin RH - Central nervous system infection in the chronically immunosuppressed. Medicine 61:166, 1982.

23. Howard RJ, Simmons RL, Najarian JS - Fungal infections in renal transplant recipients. Ann Surg 188:598, 1978.

24. Kauffman CA, Frame PT - Bone marrow toxicity associated with 5-fluorocytosine therapy. Antimicrob Agents Chemother $11: 244,1977$.

25. Littman ML - Cryptococcosis (Torulosis): current concepts and therapy. Am J Med $27: 976,1959$.

26. Littman ML, WaIter Je - Cryptococcosis: current status. Am J Med 45:922, 1968.

27. McChesney JA, Marquardt JF - Hypokalemic paralysis induced by amphotericin B. JAMA 189:1029, 1964.

28. Medoff G, Comfort M, Kobayashi GS - Synergistic action of amphotericin B and 5-fluorocytosine against yeast-like organisms. Proc Soc Exp Biol 138:571, 1971. 
29. Medoff, Kobayashi GS, Kwan CN, Schlessinger D, Venkov P - Potentiation of rifampicir and 5-fluorocytosine as antifungal antibiotics by amphotericin $B$. Proc Nat Acad Sci $69: 196,1972$.

30. Meunier-Carpentier F - Cryptococcal meningitis: a case report and review of diagnostıc procedures and therapy. Acta Clin Belg 36:360, 1981.

31. Miller RP, Bates JH - Amphotericin B toxicity: a follow-up report of 53 patients. Ann Intern Med 71:1089, 1969.

32. Nóbrega JPS, Livramento JA, Machado LR, Spina-França A - Criptococose do sistema nervoso central: avaliação da terapêtica por anfotericina-B, 5-fluorocitosina e miconazole em 18 casos. Arq Neuro-Psiquiat (Sāo Paulo) 37:28, 1979.

33. Nóbrega JPS, Livramento JA, Spina-França A - 5-fluorocitosina e anfotericina-B no tratamento da criptococose do sistema nervoso central. Arq Neuro-Psiquiat (São Paulo) $33: 210,1975$.

34. Rifkind D, Marchioro TL, Schneck SA, Hill RB Jr - Systemic fungal infections complicating renal transplantation and immunosupressive therapy: clinical, microbiologic, neurologic and pathologic features. Am J Med 43:28, 1967.

35. Sabbaga E - Micoses por fungos oportunistas nos transplantes renais. In Lacaz CS (ed): Infecções por Agentes Oportunistas, Ed Univ S Paulo, São Faulo, 1977, pః 93.

36. Sarosi GA, Parker JD, Doto IL, Tosh FE - Amphotericin B in cryptococcal meningitis: long-term results of treatment. Ann Intern Med 71:1679, 1969.

37. Schonebeck J, Polak A, Fernex M, Scholer HJ - Pharmacokinetic studies on the oral antimycotic agent 5-fluorocytosine in individuals with normal and impaired kidney function. Chemotherapy (Basel) 18:321, 1973.

38. Schroter GPJ, Temple DR, Husberg BS, Weil III R, Starzl TE - Cryptococcosis after renal transplantation: report of ten cases. Surgery $79: 268,1976$.

39. Snider WD, Simpson DM, Nielsen S, Gold JWM, Motroka CE, Posner JB - Neurologica! complications of acquired immune deficiency syndrome: analysis of 50 patients. Ann Neurol 16:403, 1983.

40. Spickard A, Butler WT, Andriole V, Utz JP - The improved prognosis of cryptococcal meningitis with amphotericin B therapy. Ann Intern Med 58:66, 1963.

41. Spina-França A - Terapêtica das neuromicoses oportunísticas. In Lacaz CS (ed): Infeccões por Agentes Oportunistas. Ed Univ S Paulo, São Paulo, 1977, pg 155.

42. Symers WStC - Amphotericin pharmacophobia. Br Med J 4:460, 1973.

43. Turcotte JG - Infection and renal transplantation. Surg Clin N Am 52:1501, 1972.

44. Utz JP - Current and future chemotherapy of central nervous system fungal infections. In Thompson RA, Green JR (eds): Advances in Neurology, 6:127, Raven, New York, 1974.

45. Utz JP - New drugs for the systemic mycoses: flucytosine and clotrimazole. Bull $N$ Acad Med $51: 1103,1975$.

46. Utz JP, Garriques IL, Sande MA, Warner JF, Mandell GL, McGehec PF, Duma RJ, Shadomy $\mathbf{S}$ - Therapy of cryptococcosis with a combination of flucytosine and amphotericin B. J Infect Dis $132: 368,1975$.

47. Weenink HR, Bruyn GW - Cryptococcosis of the nervous system. In Vinken PJ, Bruyn GW (cds): Handbook of Clinical Neurology, 35:459. North-Holland, Amsterỏam, 1978.

48. Yoshikawa TT, Fujita N, Grinnell V, Edwards JE, Feldman RA - Management of central nervous system cryptococcosis, West Med J 132:123, 1980.

49. Zylstra W - Cryptococcosis and 5-fluorocytosine, Austr N Z J Med 4:296, 1974. 